\title{
EnzMet for Versatile, Highly Sensitive Light and Electron Microscopy Staining
}

\author{
W. Liu, ${ }^{*}$ D. Mitra, * V. N. Joshi, ${ }^{*}$ R. D. Powell, ${ }^{*}$ J. F. Hainfeld,* J. L. Serrano-Velez, ** I. I. Torres- \\ Vazquez,** E. Rosa-Molinar, ${ }^{* *}$ and P. M. Takvorian*** \\ * Nanoprobes, Incorporated, Yaphank, NY 11980 \\ ** Biological Imaging Group, University of Puerto Rico-Rio Piedras, San Juan, PR 00931 \\ *** Department of Biological Sciences, Rutgers University, Newark, NJ 07102
}

EnzMet (enzyme metallography) is a staining and detection method comprising a peroxidase probe developed with a metallographic silver substrate. The dense, black signal is readily observed by light microscopy, and the electron-dense, particulate product clearly visualized in the electron microscope (EM) [1]. Background is virtually zero and diffusion is negligible. EnzMet gives clear enumeration of gene copies in in situ hybridization [2] and highly differentiated, permanent staining in immunohistochemistry [3]. Because organic chromogens such as diaminobenzidine (DAB) are used mostly with contrast enhancers such as nickel (II) [4], or with polymerized peroxidase probes to amplify the signal [5], a comparison with these methods was undertaken. Paraffin-embedded bladder carcinoma sections from the same series were stained in parallel using AE1/AE3 primary monoclonal antibody and peroxidase secondary. EnzMet using a monomeric peroxidase probe was compared with standard $\mathrm{DAB}$ and with $\mathrm{DAB}$ with $\mathrm{Ni}$ (II) enhancement using the same probe, and with DAB using a polymerized secondary probe (Envision, Dako). EnzMet gave higher sensitivity and contrast than others (Figure 1), and comparable results with up to 50-fold lower primary antibody concentrations.

EM applications of EnzMet [1] were extended to serial block-face scanning electron microscopy (SBFSEM) [6]. EnzMet immunohistochemistry combined with heavy atom staining was used to visualize Alexa Fluor ${ }^{\circledR} 594$ biocytin (Invitrogen) retrograde labeled neurons within the Western Mosquitofish (Gambusia affinis) spinal cord. EnzMet provides high contrast at neuronal boundaries, allowing contouring and three-dimensional (3D) renderings from each serial resin-embedded blockface image (Figure 2). EnzMet was also used for on-section transmission EM immunolabeling in microsoporida, protozoans responsible for opportunistic infections in immunocompromised patients, and with HIV and tropical diseases [7]. Thin sections were stained using primary anti-Ec6 antibody ( $1: 100$ dilution, 1 hour at $\left.32^{\circ} \mathrm{C}\right)$, washed with $0.2 \%$ Tween 20 - phosphate buffered saline (TPBS), incubated with 1:50 diluted peroxidase secondary $\left(1\right.$ hour at $\left.32^{\circ} \mathrm{C}\right)$, rinsed with $\mathrm{TPBS}$, deionized water and 0.1 M sodium citrate buffer, $\mathrm{pH} \mathrm{3.8,} \mathrm{then} \mathrm{developed} \mathrm{with} \mathrm{EnzMet} \mathrm{substrate} \mathrm{optimized} \mathrm{for}$ EM using a protective colloid. Dense, specific staining resulted, with uniform spherical particle morphology (Figure 3). EnzMet also gives higher contrast than DAB in Western blots (Figure 4A) and lower background than silver or gold-enhanced Nanogold in immunodot blots (Figure 4B).

References

[1] R. Powell et al., Microsc. Microanal. 12 (Suppl. 2) (2006) 424CD.

[2] R. D. Powell et al., Hum. Pathol., 38 (2007) 1145.

[3] R. Tubbs et al., Appl. Immunohistochem. Mol. Morphol., 13 (2005) 371.

[4] J. C. Adams, J. Histochem. Cytochem., 29 (1981) 775.

[5] K. H. Wiedorn et al., J. Histochem. Cytochem., 49 (2001) 1067.

[6] W. Denk and H. Horstmann, PLoS Biology, 2/e329 (2004) 1900.

[7] A. Cali et al., J. Euk. Microbiol., 57 (2010) 562. 
[8] Partly supported by NIGMS (NIH) under SBIR grant GM064257; NIMH (NIH) under SBIR grant MH086994; and by NIAID (NIH) under Research grant AI31788.

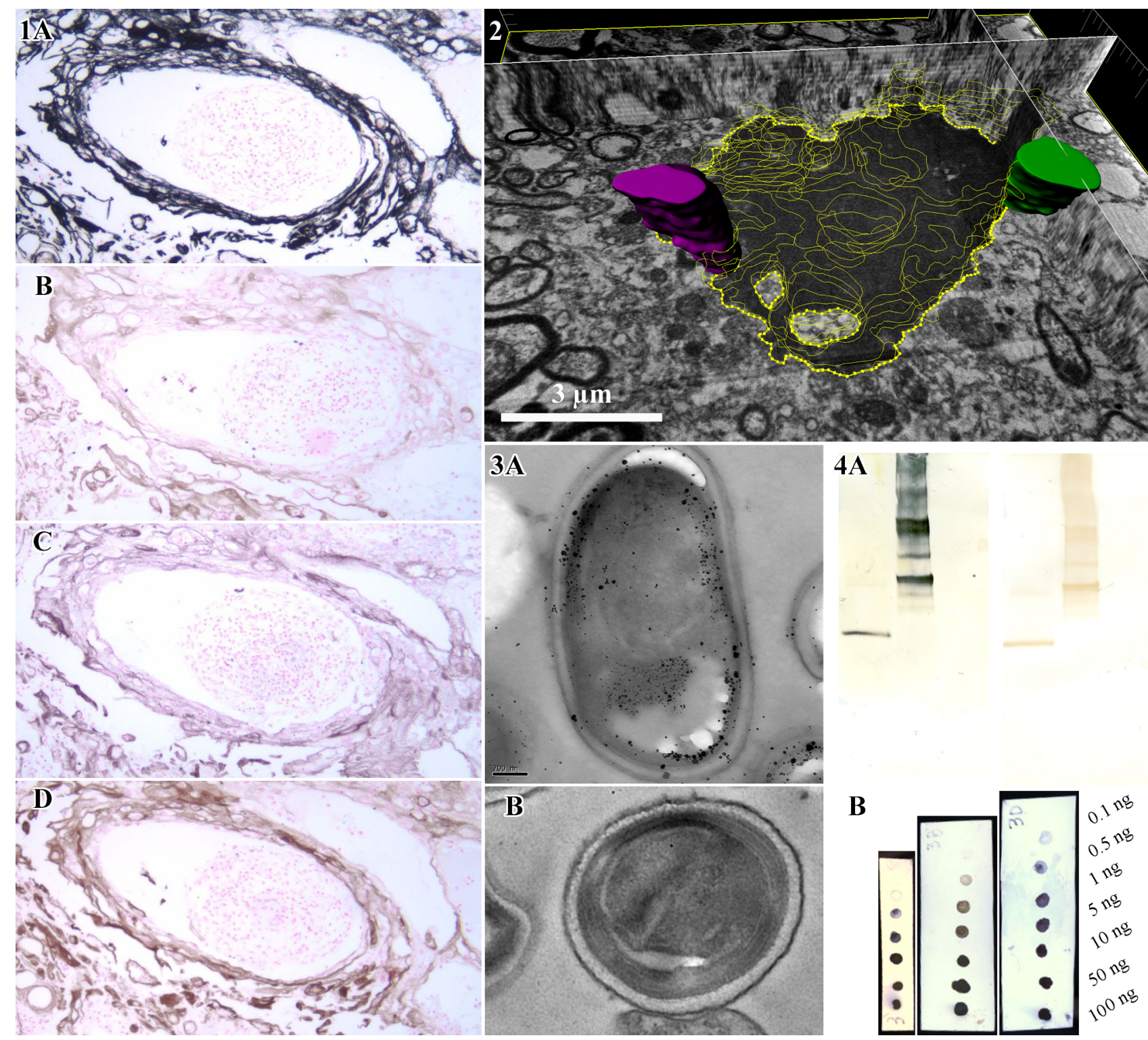

Figure 1: EnzMet vs. enhanced DAB. Cytokeratins in paraffin-embedded bladder tumor stained with monoclonal primary antibody (AE1/AE3, Dako) and peroxidase secondary with (A) EnzMet; (B) DAB; (C) DAB / Ni (II); and (D) Polymerized HRP-secondary (Envision, Dako) with DAB.

Figure 2: Serial block-face SEM: Alexa Fluor ${ }^{\circledR} 594$ biocytin retrograde labeling with EnzMet IHC and heavy atom staining, showing contouring (yellow) and 3D rendering (magenta, green) of axons. Serial resin-embedded block-face images $(n=75$; voxel size: $\mathrm{x}=0.014771, \mathrm{y}=0.014771, \mathrm{z}=0.05 \mu)$.

Figure 3: Electron microscopy on-section immunolabeling in microsporida: (A) labeling of cell wall protein Ec6 in E. cuniculi; (B) control with primary antibody omitted (bar $=200 \mathrm{~nm})$.

Figure 4: (A) Western blot detection of $100 \mathrm{ng}$ His-tagged ATF-1 (34 kD; left lane), $100 \mathrm{ng}$ Histagged YY1 (68 kD; center) and $100 \mathrm{ng}$ BSA and $100 \mathrm{ng}$ ovalbumin (control; right lane) using (left) EnzMet and (right) DAB. (B) Immunodot blot with (left) EnzMet, (center) silver and (right) goldenhanced Nanogold; note cleaner background with EnzMet. 